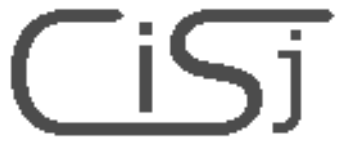

\title{
COLORED LIGHT-TO-VOLTAGE CONVERTERS BASED ABSORBANCE METER
}

\author{
M. Pereira ${ }^{1}$, O. Postolache ${ }^{1,2}$, P. Girão ${ }^{2}$, Helena Ramos ${ }^{2}$ \\ 1.Escola Superior de Tecnologia de Setúbal, Instituto Politécnico de Setúbal, 2914-508 Setúbal, Portugal, \\ 2. Instituto de Telecomunicações, DEEC, IST, Av. Rovisco Pais, 1049-001, Lisboa, Portugal \\ E-mails.joseper@est.ips.pt,poctav@alfa.ist.utl.pt,psgirao@alfa.ist.utl.pt,hgramos@alfa.ist.utl.pt
}

\begin{abstract}
A low-cost absorbance meter for dissolved compounds identification and concentration evaluation is proposed. The main elements of the system are a white light source and a set of three colored light-to-voltage converters. The system includes auto-calibration capabilities and compensates spectral sensitivity variation of the optical source and detectors. A neural network-processing scheme is used to identify dissolved compounds and evaluate the correspondent concentrations.
\end{abstract}

Keywords: absorbance measurement, error compensation, neural networks.

\section{INTRODUCTION}

Absorbance measurements have crucial importance in all chemical processes where concentration measurement is required and, particularly, when variation of concentration over time is needed as a direct measurement of a chemical reaction velocity. For a given compound and length of the light path in the medium, absorbance has a linear dependence on concentration (Beer's law)[1].

Spectrometers are generically used for absorbance, transmittance and concentration measurements but the cost of these instruments is typically high. The present paper presents a low-cost solution with enough accuracy for industrial applications with low accuracy requirements. The solution presented takes advantage of low-cost hardware components and of signal processing performed by a programmable microcontroller (PIC 16F877)[2] and pays particular attention to several system's errors minimization like: optical power source variations, ambient lighting noise and analogue-to-digital converter errors. Autocalibration capabilities [3] and a ratiometric measurement method are used to cancel common errors of the measurement chain.

Main applications of the measurement system are in the classification and concentration evaluation of solutions, and in the interpolation of absorbance measurements.. Other potential application of the system is color measurement of the water that together with turbidity measurements [4][5] can deliver important parameters for water quality evaluation.

\section{SYSTEM DESCRIPTION}

Figure 1 represents the measurement system block diagram that includes, besides the optical part, a conditioning circuit with a programmable gain amplifier (PGA), a programmable microcontroller (PIC) and a display to represent measured values.

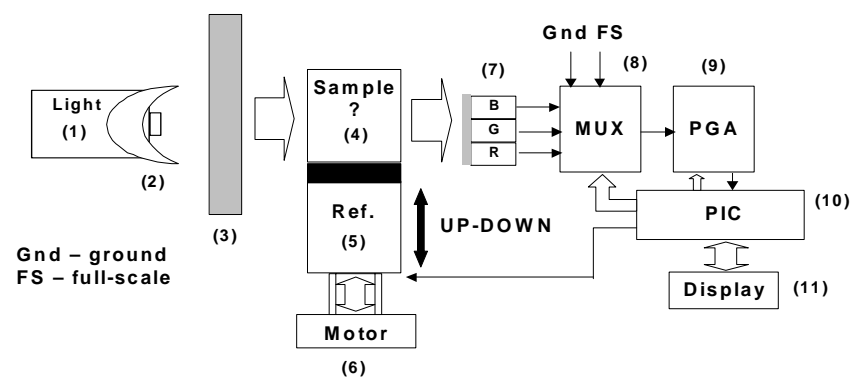

Fig. 1. Measurement system block diagram:

(1) white light source; (2) parabolic reflector; (3) optical diffuser and focusing lenses; (4) solution to be measured; (5) reference medium; (6) up-down positioning motor; (7) colored light-to-voltage converters (blue, green and red); (8) analog multiplexer; (9) programmable gain amplifier; (10) programmable interface controller; (11) display.

A two-stage measurement scheme is considered in order to overcome common errors of the measurement system. In the first stage, the sample solution is placed in front of the light beam and three colored light-tovoltage converters measure the transmitted power. In a second stage, the reference, or blank, solution is placed in front of the light beam and the transmitted 
(incident) optical power is measured. Vacuum, air, distilled water or any standard solution, with the same composition of the sample solution (comparative measurements), can be used as reference. The solution to be measured and the reference medium are placed in standard $10 \mathrm{~mm}$ path-length cells. An additional calibration procedure can be considered if a standard solution is available. In this case, the relative sensitivities of the optical detectors can be evaluated and the correspondent calibration coefficients used for error compensation. These features represent an autocalibration capability of the measurement system that improves its accuracy.

\section{A. Light Source}

The optical source selection, which affects directly the performance of every optoelectronic measurement system, is based on a compromise among the following characteristics: brightness, spectrum bandwidth, size and lifetime. The lamp and reflector chosen have the following main characteristics: 1) lamp - $5.0 \mathrm{~V}, 0.44 \mathrm{~A}, 2.2 \mathrm{~W}, 2.0$ candle power, 2750 Kelvin color temperature and 1000 life hours; 2) reflector - parabolic with 0.50 " diameter and overall length, 0.189" ream, 0.07" focal distance. Figure 2 represents the optical source spectrum that exhibits a power variation lower than $10 \mathrm{~dB}$ in the bandwidth of interest.

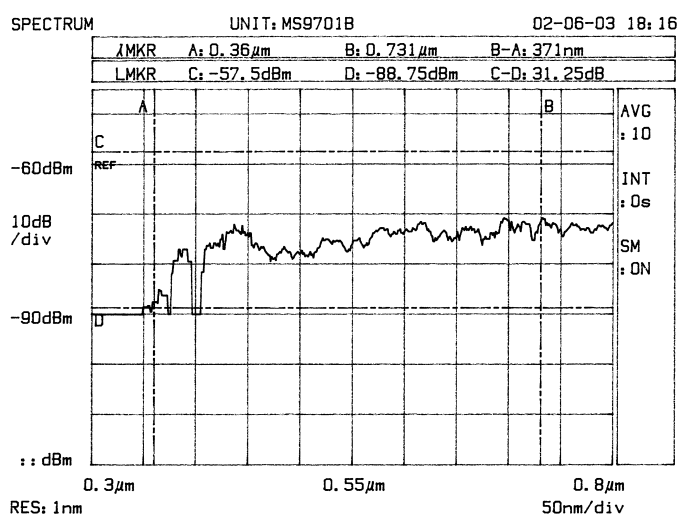

Fig. 2. Optical source spectrum.

\section{B. Detectors}

The transmitted light through the solution is detected by a set of three colored light-to-voltage converters. Figure 3 represents the electrical diagram of the selected detectors (TSLB257, TSLG257 and TSLR257). The detectors are high-sensitivity and lownoise light-to-voltage converters that include in a compact 3-leaded plastic package the following main elements: a color filter, a photodiode and a transimpedance amplifier. The output voltage is directly proportional to the filtered light intensity (irradiance), on each photodiode, and the maximum wavelength sensitivities for blue, green and red detectors is $470 \mathrm{~nm}, 565 \mathrm{~nm}$ and $635 \mathrm{~nm}$, respectively. Each device has a transimpedance gain of $320 \mathrm{M} \Omega$, a dark voltage lower than $15 \mathrm{mV}$ and an integrated r.m.s. (root mean square) noise voltage (dc to $1 \mathrm{kHz}$ ) lower than $200 \mu \mathrm{V}$. Transimpedance amplifier transfer function is given by:

$$
\mathrm{G}(\mathrm{s})=\frac{\mathrm{V}_{0}(\mathrm{~s})}{\mathrm{I}_{\mathrm{L}}(\mathrm{s})}=\frac{\mathrm{R}}{1+\mathrm{sRC}}
$$

where $\mathrm{V}_{0}$ represents the output voltage and $\mathrm{I}_{\mathrm{L}}$ the input current that is proportional to the light intensity detected by the photodiode.

Internal RC components assure a time constant of approximately $70 \mu \mathrm{s}$, which mean that the cut-off frequency of the low-pass filter is about $2.27 \mathrm{kHz}$.

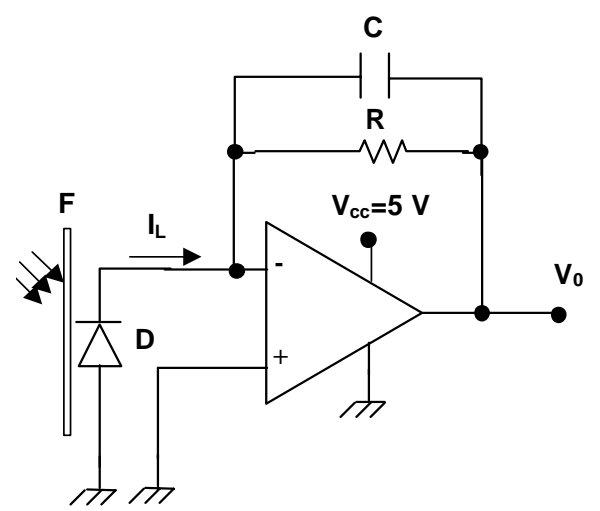

Fig. 3. Electrical diagram of the colored light-to-voltage converter integrated circuit

(F- optical filter, D- photodiode, $\mathrm{V}_{0^{-}}$output voltage, $\mathrm{V}_{\mathrm{CC}^{-}}$ unipolar power supply).

The output voltage $\left(\mathrm{V}_{0}\right)$ depends mainly on the solution absorbance and on the photodiode spectral responsitivity (Figure 4).

Considering the peak wavelength and the spectral halfwidth of each detector that are equal to: $\lambda_{1}=470$ $\mathrm{nm}, \Delta \lambda_{1 / 2}=35 \mathrm{~nm}, \lambda_{2}=565 \mathrm{~nm}, \Delta \lambda_{1 / 2}=28 \mathrm{~nm}, \quad \lambda_{3}=635$ $\mathrm{nm}, \Delta \lambda_{1 / 2}=25 \mathrm{~nm}$, and assuming that the power spectral distribution is approximated by a gaussian function, the output voltage of each photodiode is given by:

$$
\mathrm{V}_{\mathrm{i}} \cong \int_{\lambda \min _{\mathrm{i}}}^{\lambda \mathrm{max}_{\mathrm{i}}}(\lambda) \mathrm{g}(\lambda) \mathrm{d} \lambda
$$


where $f_{i}$ represents the spectral responsitivity of each photodiode, $\mathrm{g}$ the solution absorbance, and $\lambda \min _{\mathrm{i}}$ and $\lambda \max _{\mathrm{i}}$ are the wavelengths associated with the bandwidth of each photodiode.

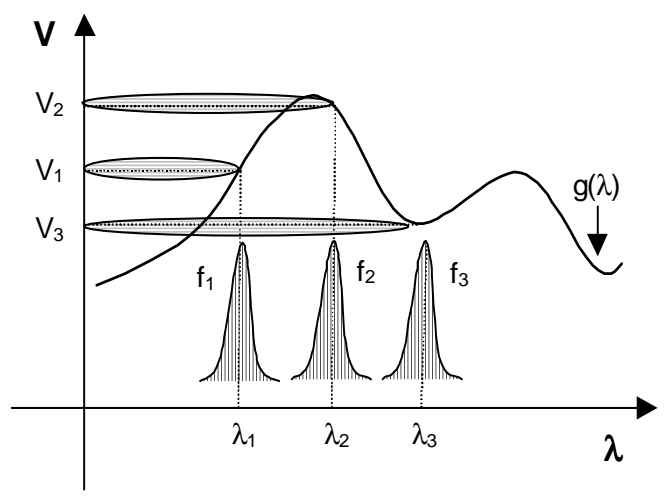

Fig. 4. Detector output voltage as a function of solution absorbance and photodiode spectral responsitivity $\left(f_{1}\right.$ blue detector, $f_{2}$ - green detector, $f_{3}$ - red detector).

\section{Signal Conditioning}

Signal conditioning [6] includes a PGA whose gain is automatically adjusted according to its input voltage level in order to minimize relative errors that are specially important when solution absorbance is high. A ratiometric measurement of incident and transmitted optical powers makes system's accuracy independent of source intensity variations and other common errors of the measurement chain, like temperature and ambient light.

Offset and gain errors of PIC's analog-to-digital converter (ADC) are also cancelled by using ground and full-scale (FS) amplitude measurements.

Figure 5 represents the schematic diagram of the conditioning circuit that includes an instrumentation amplifier (AD524) whose gains of 10-100-1000 are automatically selected by the PIC.

If $V_{M}$ and $V_{R}$ represent the voltages that come from the sample solution and from the reference medium, the absorbance for a given wavelength $(\lambda)$ is given by:

$$
\mathrm{A}(\lambda)=\log _{10}\left(\frac{\mathrm{P}_{0}}{\mathrm{P}}\right)=\log _{10}\left(\frac{\mathrm{V}_{\mathrm{R}} / \mathrm{G}_{\mathrm{R}}}{\mathrm{V}_{\mathrm{M}} / \mathrm{G}_{\mathrm{M}}}\right)
$$

where $\mathrm{P}_{0}$ is the optical incident power, $\mathrm{P}$ is the optical transmitted power through the sample solution, $G_{M}$ and $G_{R}$ represent the selected gain for sample and reference medium measurements, respectively.

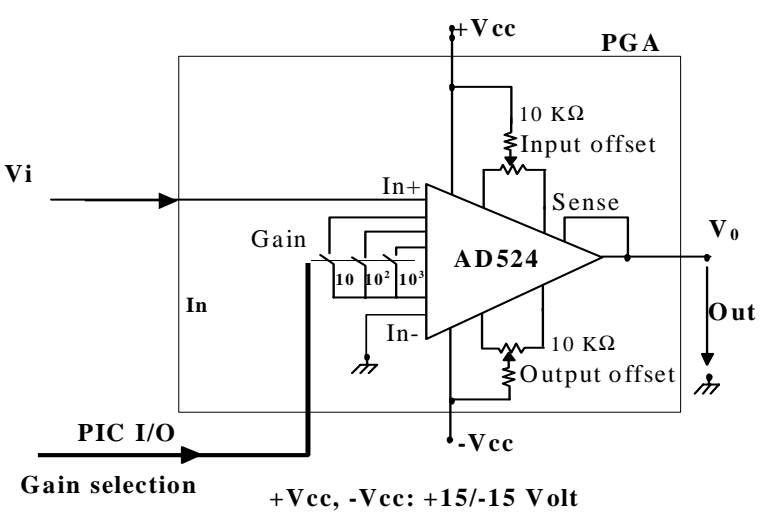

Fig. 5. PGA conditioning circuit (AD524instrumentation amplifier with selectable gain).

For transmittance measurements, the output voltages from the light converters are acquired and the ratio between transmitted and incident power is computed to provide the transmittance measurement. In this case, the transmittance for a given wavelength $(\lambda)$ is given by:

$$
\mathrm{T}(\lambda)=\left(\frac{\mathrm{P}}{\mathrm{P}_{0}}\right)=10^{-\mathrm{abc}}=\left(\frac{\mathrm{V}_{\mathrm{M}} / \mathrm{G}_{\mathrm{M}}}{\mathrm{V}_{\mathrm{R}} / \mathrm{G}_{\mathrm{R}}}\right)
$$

where a represents the absortivity of the dissolved compound, $\underline{b}$ the length of the absorption cell (10 $\mathrm{mm})$, c the compound concentration and the others variables have the meanings previously defined.

\section{ANN PROCESSING}

Signal processing is required to extract the required information from sensors output voltages. Considering the typical non-linear response of the detectors (Figure 4) a neural processing structure [7-9] with three neural networks is considered: one network is used to classify the solution (NN classif), another is used to obtain the tested solution concentration (NN conc.) and the third is used to obtain the absorbance behavior of the tested solution for different wavelenghts ( $\mathrm{NN}$ abs) (Figure 6). The structure implemented exhibits a good performance even when training of the individual networks uses low size data sets [10].

Besides the general advantages of the usage of neural networks [11], in this particular case their capacity to pattern recognition is used to identify the type of the solution and their capacity to model nonlinear systems behavior (Figure 7) is used to interpolate and extrapolate absorbance measurement data. 


\section{A. Solution Classification (Pattern Recognition)}

As mentioned before, one of the objectives of the measuring system is to allow the classification of solutions based on their absorbance at three different wavelengths (pattern recognition).

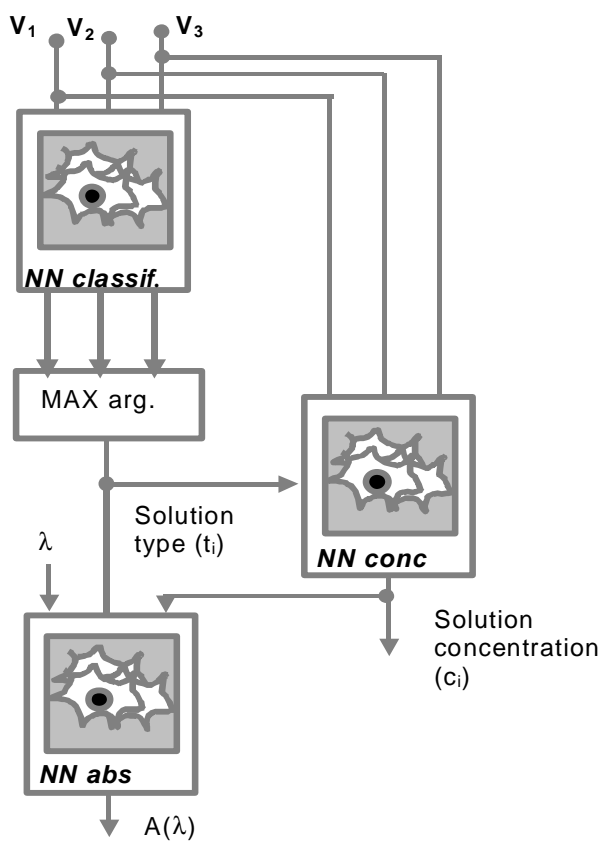

Fig. 6. NN processing structure.

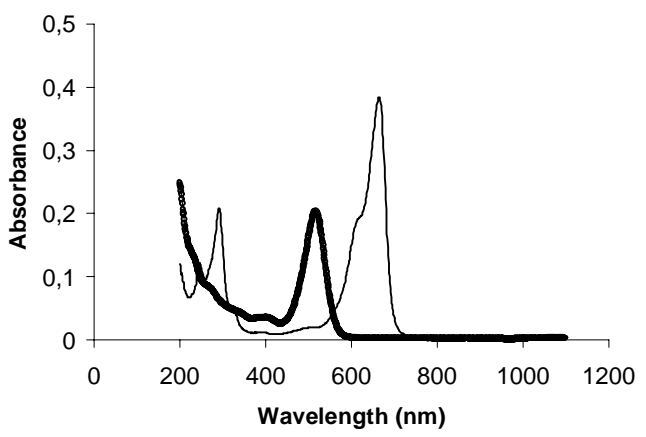

Fig. 7. Absorbance results of methylene blue (normal line) and eosin blueish (bold line) solutions with concentrations equal to 5E-6 $\mathrm{M}$.

To exemplify let us consider that the solution to classify belongs to a universe of three solution types (e.g.. methylene blue, eosin blueish and eosin yellowish), as depicted in Figure 6 (NN classif). Then, a three input, three-output multiplayer perceptron NN can be used. Referring to the training set, it includes the normalized values of the acquired voltage of TSLB257 (blue sensor), TSLG257 (green sensor) and
TSLR257 (red sensor) for the three detectors and for different concentrations of each solution $(5 \mathrm{E}-5 \mathrm{M}, 1 \mathrm{E}-$ $6 \mathrm{M}, 1 \mathrm{E}-7 \mathrm{M}, 1 \mathrm{E}-8 \mathrm{M})$. Thus, for the NN classif, the input matrix of the training set is expressed by light sensor delivered voltages and the output matrix is expressed by the solution type identified by binary sequence with a single 1 value. The $\mathrm{NN}$ target matrix is then expressed by:

$$
\mathrm{T}_{\text {classif }}=\left[\begin{array}{lll}
1 & 0 & 0 \\
0 & 1 & 0 \\
0 & 0 & 1
\end{array}\right]
$$

The used NN classif characteristics are: input neurons: 3, hidden neurons: 5 tansignoid, output neurons: 3 linear. The training stop condition is $\mathrm{SSE}_{\mathrm{STOP}}<0.1$.

The obtained NN output values are applied to MAX arg block that selects the type of solution based on the maximum value of the $\mathrm{NN}$ output vector. Thus, considering the NN classif test vector $\mathbf{V}_{\mathbf{t}}=\left[\begin{array}{ll}\mathbf{v}_{1} & \mathrm{v}_{2} \\ \mathrm{v}_{3}\end{array}\right]^{\mathrm{T}}$, and the corresponding NN output vector $\mathbf{O}_{t}=\left[\begin{array}{lll}t_{1} & t_{2} & t_{3}\end{array}\right]^{T}$ the solution identification is based on the following relations:

$$
\begin{array}{ccc}
\text { solution } 1 & \text { if } \quad t_{1}=\max _{i=1 \ldots 3}\left(t_{i}\right) \text { and. }\left|t_{1}-1\right|<\delta \\
\text { solution } 2 & \text { if } \quad t_{2}=\max _{i=1 . .3}\left(t_{i}\right) \text {.and. }\left|t_{2}-1\right|<\delta \\
\text { solution } 3 & \text { if } \quad t_{3}=\max _{i=1 \ldots 3}\left(t_{i}\right) \text {.and. }\left|t_{3}-1\right|<\delta
\end{array}
$$

where $\delta=[0 ; 0.5]$ represents the identification threshold

\section{B. Concentration Evaluation}

For concentration evaluation a three layers perceptron neural network is also used. The hidden layer neurons have also tansignoid activation functions and the dependence between the number of hidden neurons and the $\mathrm{NN}$ performance is presented in Figure 8 .

Analyzing the figure it is possible to conclude that the optimal solution is 14 tansignoid neurons in the hidden layer. The error in this case is smaller than 0.05 $\mathrm{ppm}$. As output layer is used one linear neuron that delivers the concentration information. The set used to train the network is the same used to train NN classif. After the training phase, the $\mathrm{NN}$ is tested using different solutions with concentrations included in the $[0.01,50] \mathrm{ppm}$ interval. 


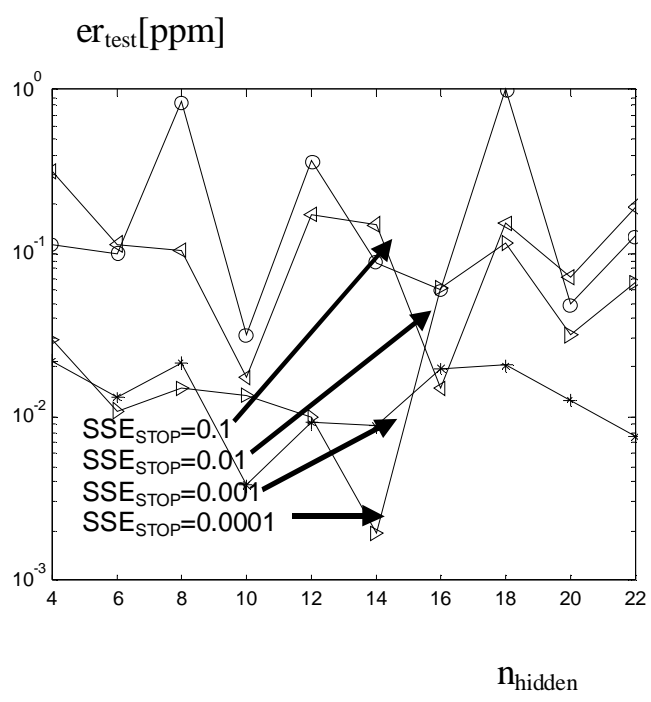

Fig. 8. The evolution of $\mathrm{NN}$ conc test error versus the SSE training stop condition and the number of neurons on the hidden layer.

\section{Absorbance Characteristic Evaluation}

The interpolation and extrapolation capabilities of neural networks are used to obtain the absorbance values for the different type of solutions of a given concentration. Training data is obtained using a spectrometer UNICAM 5625 series. The NN used (NN abs) has three inputs and one output perceptron. The number of hidden tansignoid neurons is equal to 10 and the training stop condition is expressed by $\mathrm{SSE}_{\mathrm{STOP}}<1 \mathrm{E}-4$.

\section{RESULTS}

\section{A. Solution Classification}

Several results regarding solution classification are presented in Figure 9 under the form of detection rate (DR) versus threshold value, $\delta$. The results are experimental and obtained for different test sets. The detection rate (DR) is calculated using the following formula:

$$
\operatorname{DR}[\%]=\operatorname{abs}\left(1-\frac{\mathrm{n}_{\text {real }}-\mathrm{n}_{\text {det ect }}}{\mathrm{n}_{\text {real }}}\right) \cdot 100
$$

where $\mathrm{n}_{\text {real }}$ is the number of values for classification and $\mathrm{n}_{\text {detect }}$ is the number of correct classifications.

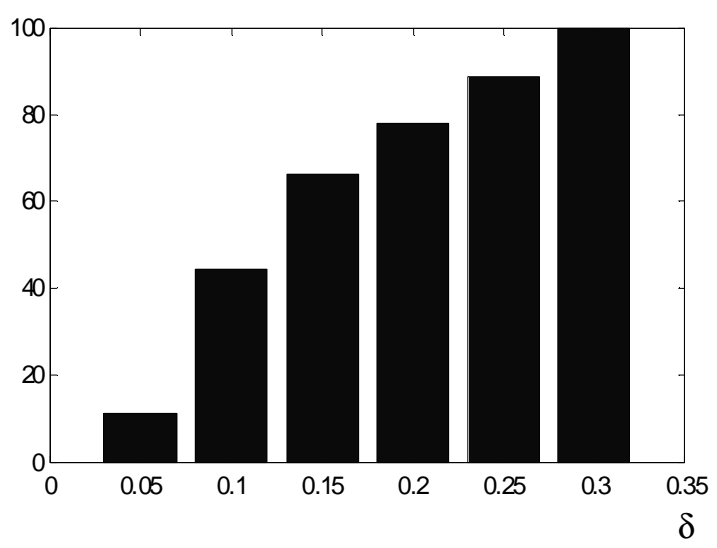

Fig. 9. Solution classification: DR versus $\delta$.

Considering previous results a threshold of 0.3 is used in order to maximize detection rate.

\section{B. Concentration Evaluation}

As shown in Figure 8 for a particular type of solution (eosin blueish), for the selected number of hidden neurons (14) and stop condition (1E-4), the error in concentration evaluation using the measuring system is $2 \times 10^{-3} \mathrm{ppm}$ in the worst case. This value corresponds to relative errors much smaller than $1 \%$. Thus, it is fairly safe to say that the system allows concentration measurements with $1 \%$ inaccuracy.

\section{Absorbance Evaluation}

Considering that the solution type and concentration are known, Beer's law establishes, for low solution concentrations (c<1E-2 M), a linear dependence between absorbance and concentration:

$$
\mathrm{A}(\lambda)=\varepsilon(\lambda) \mathrm{bc}
$$

where $\mathrm{A}$ is the absorbance, $\varepsilon$ the absortivity of the dissolved compound, b the optical cell length and $c$ solution's concentration. Theoreticaly in a logarithmic scale, the curves of absorbance for different concentrations can be obtained by translation. However practical results shows significant deviations from Beer's law even for low concentrations. Figure 10 represents absorbances results for an eosin blueish solution with the following concentrations: $\mathrm{S} 1-5.10^{-5}$ $\mathrm{M} ; \mathrm{S} 2-10^{-6} \mathrm{M} ; \mathrm{S} 3-10^{-7} \mathrm{M}$; S4 - $10^{-8} \mathrm{M}$. 


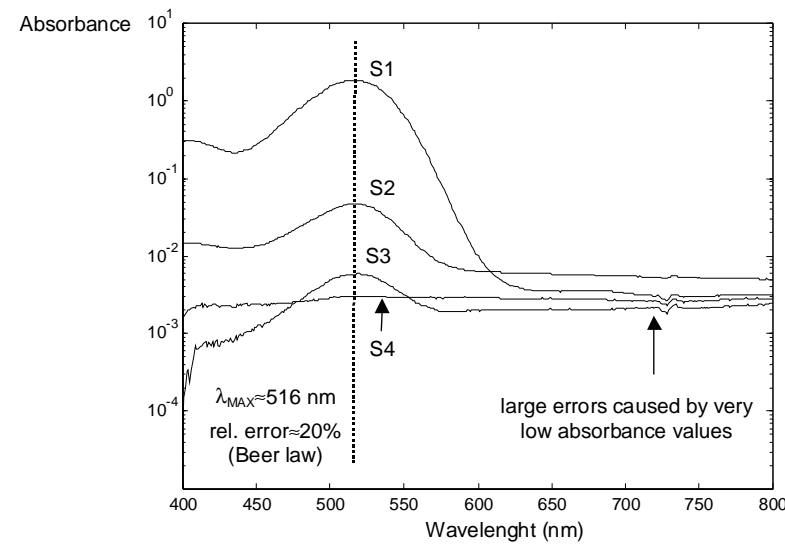

Fig. 10. Beer's law deviations: absorbance for different values of concentrations.

To test the interpolation capabilities of the neural network ( $\mathrm{NN}$ abs) in foreseeing the absorbance results of a solution of a given concentration, two solutions of eosin blueish with different concentrations values are considered, $\mathrm{C} 1=5 \mathrm{E}-5 \mathrm{M}$ and $\mathrm{C} 2=5 \mathrm{E}-6 \mathrm{M}$, and the correspondent spectrometer data is used to train the $\mathrm{NN}$ abs. After the training phase, the $\mathrm{NN}$ abs is tested using eosin blueish solution characterized by $\mathrm{C} 3=(\mathrm{C} 1+\mathrm{C} 2) / 2$. The normalized absorbance evolution obtained in the test phase for the three concentrations is shown in Figure 11.

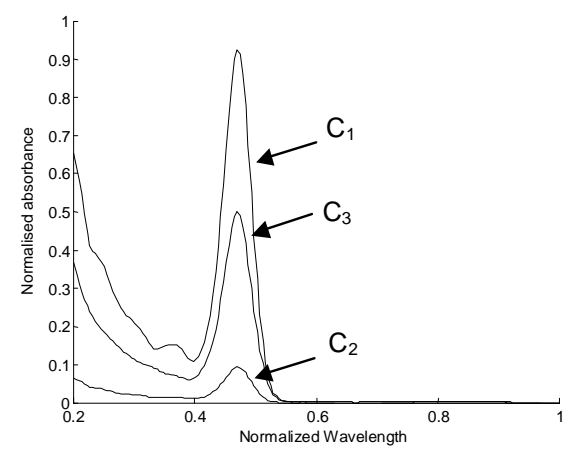

Fig.11. The normalized absorbance graph obtained on the NN abs test phase.

Referring to the results obtained for $\mathrm{C} 3$ using the $\mathrm{NN}$ abs, the absorbance evaluation relative error is calculated using data obtained with the spectrometer. Error results are presented in Figure 12 that reveals a relative errors smaller than $3 \%$. It should be noted that since the absorvity is a function of the wavelength, it is only possible to obtain the curve of $\mathrm{C} 3$ from curves relative to $\mathrm{C} 1$ and $\mathrm{C} 2$ if that dependence in known.

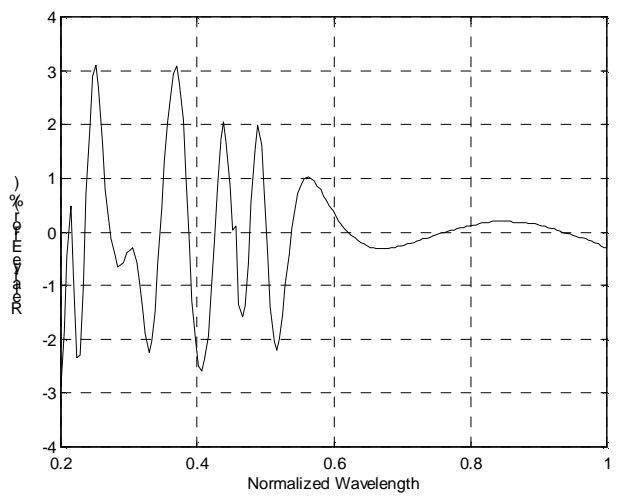

Fig.12. The absorbance evaluation relative error for the NN abs and tested eosin blueish solution.

\section{CONCLUSION}

The paper presents a low-cost solution for absorbance and transmittance measurements. The main characteristics and novelties include autocalibration capabilities to compensate for incident power variations, external light disturbances, offset and gain ADC errors and sensitivity variation of optical detectors (aging). Ratiometric measurements and a post-processing software, based on artificial neural networks, improves system's accuracy and provides identification of sample compounds and correspondent concentrations.

In what solution classification is concerned, the proposed measuring system produces total success provided that a convenient design is used. In concentration and absorbance evaluation, the errors obtained with the system are smaller than $1 \%$ and $3 \%$, respectively, values quite acceptable for industrial applications requiring medium accuracy.

\section{ACKNOWLEDGEMENTS}

This work was supported both by Portuguese Science and Technology Foundation PRAXIS XXI program FCT/BPD/2203/99 and by Project FCT PNAT/1999/EEI/15052. These supports are gratefully acknowledged. We also would like to thank the Departamento de Sistemas e Informática da ESTSetúbal, IPS, for their important technical support.

\section{REFERENCES}

[1] D. Skoog, F.J. Holler, T.A. Nieman, Principles of Instrumental Analysis, $5^{\text {th }}$ edition, Saunders College Publishing, 1998.

[2] John B. Peatman, Design with PIC Microcontrollers, Prentice Hall, 1998. 
[3] Frank van der Goes, Gerard Meijer, "A Simple Accurate Bridge-Transducer Interface with Continuous Autocalibration", IEEE Trans. Instrum. Meas., Vol. 46, No.3, pp. 704-710, June 1997.

[4] O. Postolache, M. Pereira, P. Girão, "An Intelligent Turbidity and Temperature Sensing Unit for Water Quality Assessment", Proc. of 2002 IEEE Canadian Conference on Electrical \& Computer Engineering, Winnipeg, Canada, 12-15 May 2002, pp.494-499.

[5]O. Postolache, P. Girão, M. Pereira, H. Ramos, An IR Turbidity Sensor: Design and Application, Proc. of IEEE Instrumentation and Measurement Technology Conference, Anchorage, AK, USA, 21 23 May 2002, pp.535-540.

[6] Daniel H. Sheingold, Transducer Interfacing Handbook, Analog Devices Inc., 1981.

[7] R. Rojas, Neural Networks - A Systematic Introduction, Springer Berlin Heidelberg, New York, 1996.

[8] S. Haykin, Neural Networks - A Comprehensive Foundation, Prentice Hall, 1999.

[9] C. Jain, N. M. Martin, Fusion of Neural Networks, Fuzzy Seta, and Genetic Algorithms - Industrial Applcations, CRC Press LLC, 1998.

[10] J.M. Dias Pereira, P. Silva Girão, O. Postolache, "Fitting Transducer Characteristics to Measured Data”, IEEE Instrumentation \& Measurement Magazine, Vol.4, No.4, pp. 26-39, Dec. 2001.

[11] A. Bernieri, G. Betta, A. Pietrosanto and C. Sansone "A neural network approach to instrument fault detection and isolation" IEEE Trans.Instr. Meas., vol. 44, pp.747-750, June 1997.

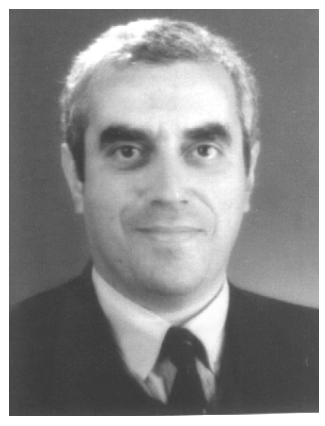

J.M. Dias Pereira (M'02) received his degree in Electrical Engineering from the Instituto Superior Técnico (IST) of the Technical University of Lisbon (UTL) in 1982. He worked for eight years for Portugal Telecom in digital switching and transmission systems. In 1992, he returned to teaching as Assistant Professor in Escola Superior de Tecnologia of Instituto Politécnico de Setúbal, where he is, at present, a Coordinator Professor. In 1995, he received the MSc degree and in 1999 the PhD degree in Electrical Engineering and Computer Science from IST. His main research interests are in the instrumentation and measurements areas.

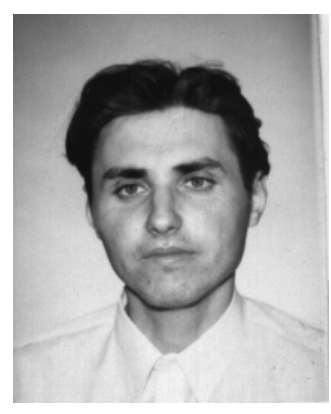

Octavian Postolache (M'99) was born in Piatra Neamt, Romania, on July 29, 1967. He received the electrical engineering diploma from Technical University of lasi, Faculty of Electrical Engineering in 1992. In 1992, he joined the Faculty of Electrical Engineering lasi, Department of Electrical Measurements as an Assistant Professor where he is currently Aux. Prof.. In the last two years he develop a research activity on Instituto Superior Tecnico of Lisbon. His main research interests concern on intelligent sensor, laser systems and neural processing in automated measurement systems.

\section{P.M.B. Silva Girão (M'00,} SM'01) was born in Lisbon, Portugal, on February 27, 1952. He received the Ph.D. degree in electrical engineering from the Instituto Superior Técnico of the Technical University of Lisbon (IST/UTL) in 1988. In 1975, he joined the Department of

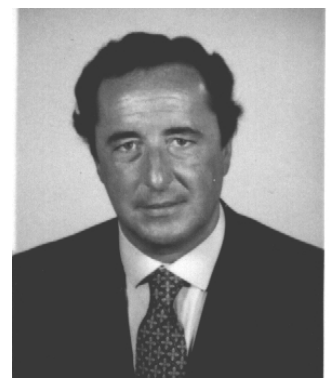
Electrical Engineering at IST/UTL, first as an Assistant Professor and, since 1988, as an Associate Professor. His main research interests concern instrumentation, measurement techniques as well as physical and mathematical problems involved in modelling magnetic materials. Metrology, quality and electromagnetic compatibility are also areas of regular activity mainly as auditor for the Portuguese Institute for Quality (IPQ).

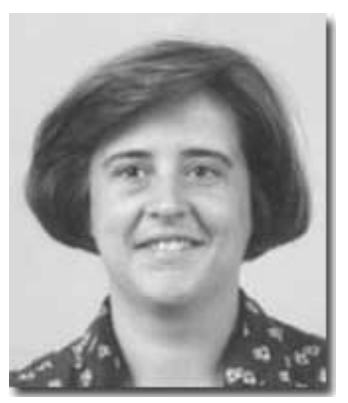

Helena Maria G. Ramos was born in Lisbon, Portugal, on October 18, 1957. She received the M.Sc. and Ph.D. degrees in electrical engineering from the Instituto Superior Técnico of the Technical University of Lisbon (IST/UTL), in 1987 and 1995 respectively. In 1981, she joined the Department of Electrical Engineering at IST/UTL, first as Assistant and, since 1995, as a Professor. Her main research interests are in the area of instrumentation and measurement techniques. 